\title{
Los Conocimientos Astronómicos de los Primitivos Peruanos
}

Es evidente que los antiguos peruanos tenían un conocimiento proiundo de la astronomía, pues habían llegado a determinar con toda precisión la duración del año computándolo en 365 días, y 1/4; conocían, además, las fechas precisas del comienzo de las estaciones, dato indispensable para la iniciaciọ́n de las faenas agrícolas.

Todos los pueblos primitivos, especialmente los del Asia Menor, habían llegado a conclusiones semejantes valiéndose de observaciones astronómicas; así por ejemplo los caldeos determinaban con toda precisión el comienzo de las cuatro estaciones y tenían una estrella que, en una posición determinada, marcaba dicho comienzo para cada es tación.

Esta determinada posición, observada no sólo entre los caldeos sino iambién entre los egipcios para los mismos fines, consiste en èl orto o en el ocaso heliaco de las estrellas elegidas. La creencia de que el orto u ocaso heliaco de una estrella se produce exactamente al cabo de un año, adolece de qun error débiablalalprecisión de los equinoccios, que en esa época era desconocida; así, después de un largo espacio de tiempo, el orto heliaco de una estrella que determinaba el principio de la primavera, por ejemplo, ya no corresponde a esta circunstancia.

Es lógico suponer que los antiguos peruanos, agricultores y pastores como sus coetáneos del Asia Menor, y que, como ellos, llegaron a determinar por observaciones astronómicas tanto la duración del año como el comienzo de las estaciones, se valieron de los mismos fenómenos para los mismos fines.

Si llegárämos a conocer la dirección en que se produjo el ocaso heliaco de una determinada estrella en aquella época, comparándola con la que corresponde en la actualidad al mismo fenómeno, el ángulo formado por la dos direcciones nos permitiría fácilmente deducir la edad - la época de la primera determinación. Para esto necesitaríamos 'conocer e identificar las constelaciones y las estrellas principales que los amautas utilizaban en sus observaciones astronómicas. 
Los amautas, lo mismo que los sacerdotes caldeos y egipcios, conservaban su ciencia astronómica en el más absoluto secreto. y sólo dejaban traslucir a! pueblo el resultado de sus investigaciones presentándolo como revelación divina y haciéndolo objeto de veneración y culto tanto a los astros de que se servían como a los fenómenos celestes anunciados.

Desgraciadamente los primeros cronistas que tomaron de boca de los descendientes de los amautas la descripción de las constelaciones veneradas por el puebio y que las identificaron en el cielo, se concretaron a destruir la idolatría sin profundizar en las causas de esta adoración que seguramente eran de orden científico. Como carecían de conocimientos astronómicos, tomaron unas constelaciones por otras sembrando la confusión entre los que posteriormente se han dedicado a investigar sobre este asunto, el último de los cuales, el señor R. Lehmann Nitsche ha fabricado un edificio enorme a base del error fundamental de la identificación de la estrella Urcuchillay.

\section{LA ESTRELLA Y CONSTELACION "URCUCHILlay"}

Siguiendo, como todos, el error cometido por el Licenciado Polo de Ondegardo al identificar a Urcuchillay como la estrella que "los astrólogos llaman Lira", el señor Lehmann ha querido encontrar la figura de una llama en algunas cestrellascdeledaliconstelación Lira y en las tres más próximas de la Hércules.

$\mathrm{Si}$ se toma un grupo de estrellas en una región cualquiera de un planisferio celeste y se las une dos a dos por medio de trazos convenientemente dispuestos, se puede construir todas las figuras que le dicte a uno la fantasía; esto es muy fácil y está al alcance de cualquiera; pero encontrar estas figuras en el Cielo, enlazando con líneas imaginarias estrellas hasta de $4^{a}$ magnitud, casi imperceptibles a simple vista, como en el caso del Urcuchillay de Lehmann, es cosa ya muy seria - si no imposible- y requiere una gran dosis de buena voluntad imaginativa.

La dificuliad estriba principalmente en las dos circunstancias siguientes: 1?) Los planisferios celestes representan el àspecto del cielo como si el observador estuviera lejos de la esfera celeste, contemplándola de fuera para dentro; pero en la realidad sucede lo contrario, nosotros la observamos de dentro para fuera y esto cambia totalmente la cuestión; pues la figura que se ve en el papel resulta en la realidad invertida sobre el espacio celeste. $2^{\circ}$ )—El aspecto del cielo cambia con la latitud 
del lugar de observación y, a menor abundamiento, cuando el observador se traslada de un hemisferio a otro. Así por ejemplo si un observador, mirando hacia el Sur desde el hemisferio boreal, cree ver en un grupo de estrellas zodiacales determinado objeto o animal puesto de pie, el mismo observador trasladado al hemisferio austral podrá reconocer en aquel grupo de estrellas al mismo objeto o animal que imaginó al verlo desde el otro hemisferio; pero esta vez lo verá hacia el Norte y con la cabeza pari abajo. Un observador del hemisferio austral, que no conociera el otro hemisferio (tal es el caso de los quichuas primitivos) iamás podrá imaginar en aquel grupo de estrellas zodiacales el mismo objeto o anima! que había creído vei el observador norteño; su fantasía

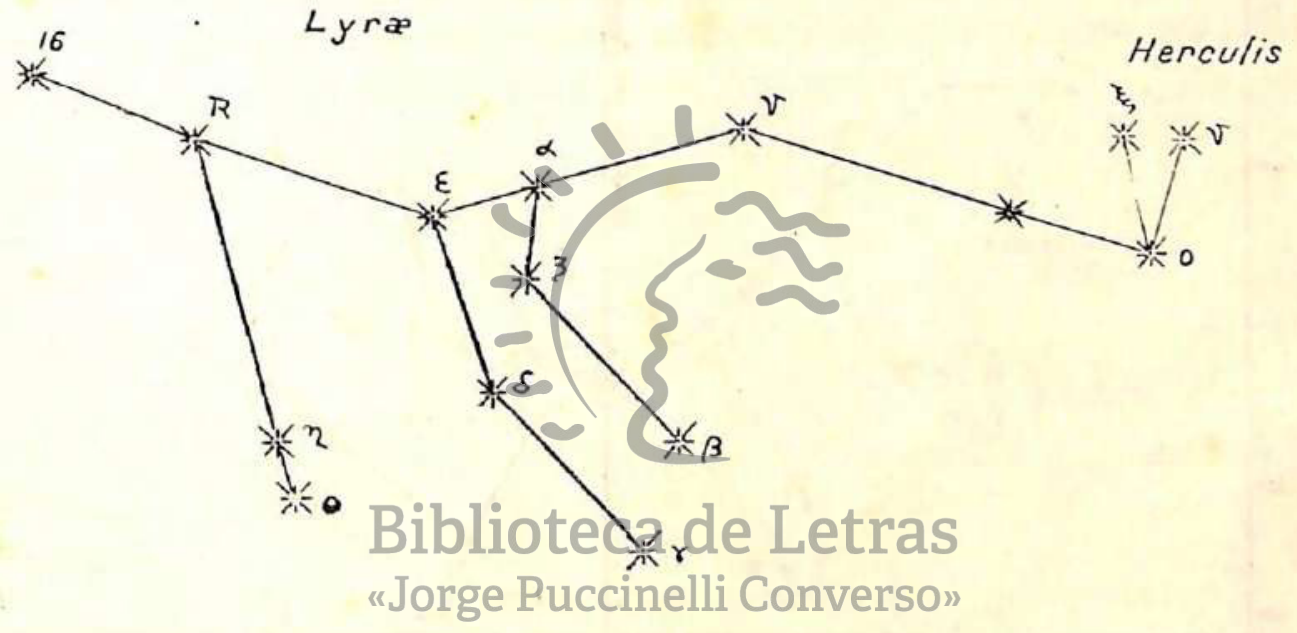

Urcuchillay, según Lehmann p. 138

Fig. 1

lo inducirá, en el mejor de los casos, a ver en aquel grupo de estrellas otra cosa muy distirita y en concordancia con sus creencias religiosas o sociales diferentes, desde luego, de las que se profesan en los pueblos ignorados del Norte.

El señor Lehınarı, sin tener en cuenta estas circunstancias, ha seleccionado once estrellas de la consteliación de lia Lira y tres de la Hércules entre las que figuran siete (nu Herculis y nu, zeta, delta, epsilón, eta y la No 16 Lirae! tan insignificantes que ni siquiera las toma en consideración las principales efemérides del mundo: American Ephemerides, Nau- 
tical Almanac, Connaissanze des Temos y Berliner Jahrbuch (FK3); ha hecho abstracción en cambio de las otras tres, tanto o más brillantes que aquellas (theta Herculis y iota y $5 B$ Lirae) y que se encuentran dentro del mismo grupo. Con estas if estrellas ha formado la figura de la página 138 de su monografía que representa, evidentemente, una llama estilizada como puede verse en la reproducción que insertamos (Fig. 1). Pero, por las consideraciones que anotamos antes, aquellas estrellas jamás se presentan en tal forma para un observador situado en el Cusco; en caso de que éste fudiera reconstruirla con un esfuerzo de imaginación y

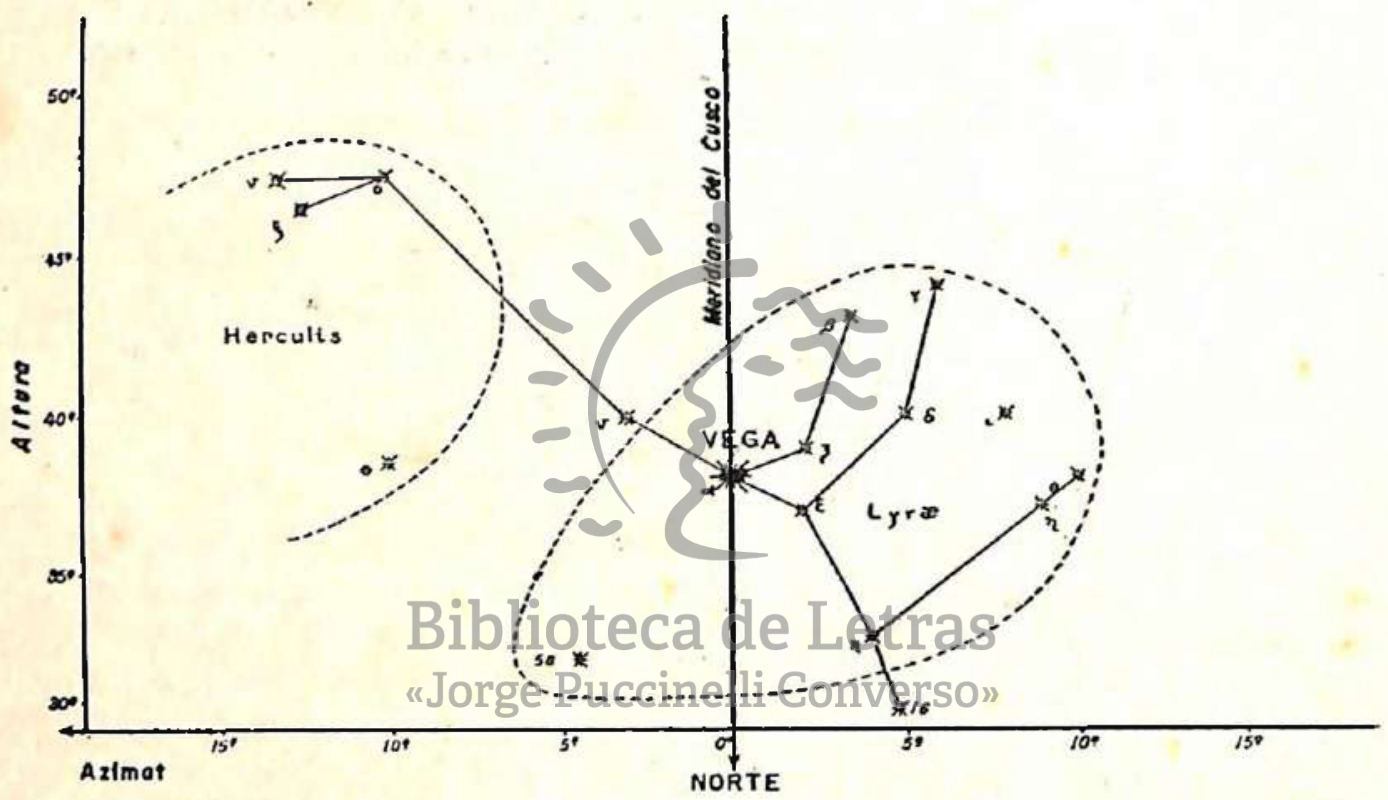

Conateilociones Lyra y Herculle vistas del Cusco en 1550, en la culminacion

d. VEGA

Fig. 2

una gran dosis de buena voluntad, la vería con las patas para arriba; Y si a esto se añade que la mayor parte de las estrellas que jalonan la silueta de aquella llama son, como ya lo dijimos, casi imperceptibles a simple vista por su pequeñez, y que, en cambio, entre ellas hay otas tanto o más brillantes, que no se les ha tomado en cuenta, no habrá ser humano que pueda encontrar en ese grupo de estrellas algo parecido a una llama Fig. 2).

Los nombres de ia mayor parte de las constelaciones son producto de la imaginación de los antiguos egipcios y caldeos, salvo muy raras 
excepciones, todos ellos han llegado hasta nosotros traducidos por los griegos y adaptados a su mitología. Así el nombre de Lira, dado a la constelación boreal yure nos ocupa, proviene de la semejanza que los egipcios creyeron encontrar entre la constelación y el perfil de una tortuga, animal que sinibolizaba el instrumento musical denominado después Lira. Al respecto, dice la leyenda que Thoth Trimegišto, dios mitológico egipcio similar al Mercurio de los griegos, encontró en las playas del Nilo una concha de tortuga con algunos tendones secos adheridos a sus bordes, los cuales, al ser pulsados con los dedos, producían sonidos musica'es armo::iosos, inventando así el instrumento musical que se construía on todos los pueblos antiguos con conchas de tortuga, así como se fabrican hoy, en nuestro altiplano, los instrumentos populares

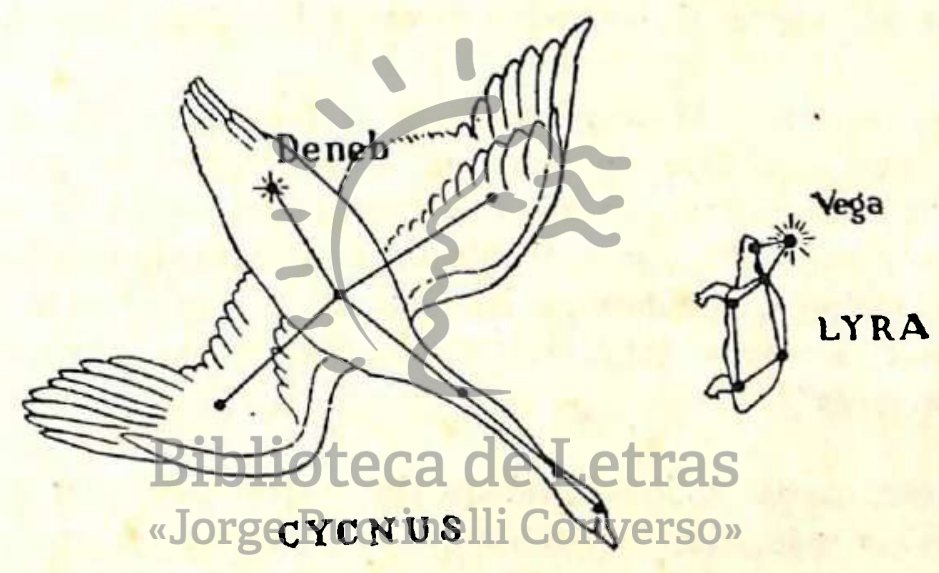

\section{Constelaciones CISNE y LIRA (Tortuga)}

Fig. 3

charango y bandurria, con las conchas de armadillo o "quirquincho". Los griegos le llamaron - tanto al instrumento de música como a la constelación-- indistintamente Lyra o Quélide, que quiere decir tortuga. Los astrónomos actuales designan la constelación por el nombre latino, generalmenle or genitivo, Lyrae y la representan por una lira de tipo moderno o por una tortuga como se ve en el grabado que reproducimos tomado del interesante artículo del Profesor Donal H. Menzel en el Nationai Geographic Magazine correspondiente a jullo de 1943 (Fig. 3). 
Ahora bien, si un determinado grupo de estrellas sugiere a priinera vista, de cualquier parte que se la mire, la idea de una tortuga, ¿cómo podømos suponer que los quichuas hubieran visto en él la forma de una llama?

Para que el lector pueda darse una idea concreta de este asunto, hemos calculado las coordenadas horizontales (distancia zenital y azimut: de las estrellas de Lira y de las tres más próximas de Hércules, sobre el horizonte del Cusco ( $13^{\circ} 5$ de latitud Sur) en el instante de la culminación de Vega (alfa Lyrae) que corresponde a las 8 de la noche, poco más o menos, de los últimos días del mes de agosto, y las hemos dispuesto en proyección mercator en el diagrama adjunto que representa aquellas estrellas para un observador situado en el Cusco o en cualquier otro lugar del gloho de aproximadamente la misma latitud. (Fig. 2).

Como se ve, sólo una imaginación fuera de todo control podría sacar de este conjunito de estrellas, representado en su aspecto real, la figura que el imalogrado señor Lehmann ha hecho "a base del cualquier mapa celestial", como él mismo lo declara, lamentándose de que "por la posición geográfica de La Plata no me es posible observar con la exactitud necesaria. esta constelación que en su época apenas asoma en el horizonte".

Por otra parte, tcdo el trabajo del señor Lehmann carece en absoluto de criterio cientifica astronómico, cosa muy" explicable porque esta rama de las ciencias no era de su especialidad como lo prueba el hecho de haber tenido que recurrir al astrónomo D. Félix Aguilar, de La Plata, para que le calculara las coordenadas ecuatoriales de Vega, así como las horas del orto y del ocaso de esta estrella sobre el horizonte del Cusco para el año 1600 más o menos.

Desgraciadamente todos los cronistas e historiadores antiguos y la mayor parte de los modernos que se han preocupado de los peruanos precolombinos adolecen del mismo defecto; por tal razón unos no le dan importancia al grado de adelanto a que hubieran podido llegar los quichuas en el estudio y aplicación de la Astronomía, y otros deliberadamente les niegan tada clase de conocimientos en esta materia.

Con lo expuests creemos haber demostrado que la constelación de la Lira no se parece a una llama por ningún lado que se la mire y, por consiguiente ella no puede ser el Urcuchillay de los quichuas. 
Entonces, ¿cuál es?

El significado del nombre y el atributo del astro (o constelación) que lo represinntaba nos van a dar la clave para llegar a él por el camino más directo.

Lehmann descompone el nombre en dos partes:

$$
\text { Urcu - chillay }
$$

$y$, luego de pretender demostrar, sin conseguirlo, que chillay es una corrupción de la palabra tilla, mal pronunciada y mal escrita, traduce:

$$
\begin{aligned}
& \text { Lircu = macho } \\
& \text { tilla = silvestre, salvaje, arisco, uraño...... }
\end{aligned}
$$

de donde deduce el siguiente significado:

Urcuchillay = Llama macho silvestre.

Vicente Fidel López descompone el nombre en tres partes:

$$
\text { Urcu - chi - illay, }
$$

cuya traducción correcta es:

$$
\begin{aligned}
& \text { Urcu = macho } \\
& \text { chi }=\text { emisión } \\
& \text { i'lay = resplandor astral }
\end{aligned}
$$

que da, sin más artificio que la contracción muy natural de chillay en chillay, el siguiente sianificado que, a mi juicio, es el verdadero:

\section{URCUCFILLAY $=$ (Llama) MACHO RESPLANDECIENTE.}

El atributo que, seqún todos los cronistas se atribuía a este astro (o constelacióni era "el ds ser ......una llama de muchos colores".

Sin ningún esfuerzo llegamos pues a dos datos fundamentales:

1.-Por el nombie:

$$
\text { Urcuchillay = Macho resplandeciente. }
$$

Resplandecientes son todos los astros, pero con diferentes intensidades. Al designar zon este nombre a una estrella determinada, se ha querido signif:car que ésta lo era en grado sumo, que era la más resplandeciente de todas 0 , por lo menos, una de las más resplandecientes.

2 - Por el atributo:

$$
\text { ".....es un carnero (llama) de muchos colores". }
$$

Adiımás de ser la más resplandeciente, más brillante o de mayor magnitud -empleancio el término científico- la estrella designada con el nombre de Utcuchillay debe dar la impresión de irradiar muchos colores. 
Estos dos datos son más que suficientes para resolver la cuestión. En efecto, sólo hay una estrella en todo el Universo que satisface a estas condiciones y ésta es Sirio, a Canis Majoris, la más brillante y la de más lindos colores.

Ninguno de los ronistas menciona a Sirio como objelo de la veneración de los antiạuos peruanos, de lo cual podría deducirse que esia estrella pasó desapercibida para ellos, cosa increible cuando, según la expresión de Puenis y Ubeda: ".....es imposible contemplar esta deslumbrante esitella sin considerar cuán misteriosas y potentes son las fuerzas de la naturaleza y sin experimentar un sentimiento profundo de admiración".

Ahora bien, jserla posible que la esritella más hermosa del Universo, la de mayor ́amaño, la de los más lindos colores, la que atraviesa el cielo del Cusco en su mayor dimensión, la que permanece visible durante mayo: tiempo $(12 \mathrm{~h}$. $30 \mathrm{~m}$.), no hubiera llamado la atención de este pueblo, eser,cialmente astrólatra?

La adora:ión de los astros tenía entre los antiguos peruanos no solamente una finalidad mística sino principalmente para la masa del pueblo, tendía a satisfacer utópicamente necesidades utilitarias terrenales, como nos lo trasmite el padre Bernabé Cobo concretando la versión de los cronistas que le precedieron:

"La adoración de las estrellas procedió de aquella opinión en que estaban de que para la conservación de Cadà especie de cosas había el Creador señalado, y como sustituído, una causa segunda; en cuya conformidad creyeron que de todos los animales y aves de la Tierra había en el Cielo un simil que atendía a la conservación y aumento dejlos, atribuyendo este oficio $y$ ministerio a varias constelaciones de estrellas".

El anima! más preciado para los indios ha sido (y lo es aún en algunos pueblos del altiplano) la llama, ya porque la utilizaban como bestia de carga o ya porque su carne constituía el principal elemento de su alimentación. En tal virtud, es lógico pensar que a este animal lo hubieran puesto bajo los auspicios del astro más grande y más hermoso, de aquel que al contemplarlo uno experimenta un sentimiento profundo de admiración, es decir de Sírio y por consiguiente de la constelación a que él perienece: el Can Mayor.

Veamos ahora el origen de los nombres Siro y Can Mayor $Y$ encontraremos una perfecta analogía en su significado con el de Urcuchillay. 
Los egipcios observaban con mucho interés a Sirio cuyo orto heiiaco les servía para determinar el comienzo del año. Sabemos que hacia el año 3.235 a.d.J.C., este fenómeno (el orto heliaco) se producía pocos días antes del $1^{\circ}$ del mes Pactuen, fecha en la que comenzaban las inundaciones del Nilo. Ellos pusieron el nombre de Perro a la constelación y - aice la 'eyenda - este perro anunciaba con sus ladridos aquellas inundaciones. A la estrella más hermosa de la constelación, la que determinaba e! comienzo del año, la llamaban Sothis que quiere decir Radiante.

Los griegos, digncs continuadores de los astrónomos egipcios y verdaderos divulgadores de la astronomía científicia, llamaron a esta Seiroios, palabra de:ivada de Seir - brillar y que significa Ardiente.

Los latinos tradujeron el nombre griego, llamándola Siruis, nombre que conserva hasta auestros días.

El nombre dado a Sirio deriva pues de su cualidad esencial de brillantez y resplandor deslumbrantes y su significado es idéntico en esencia:

\section{Pára los egipcios, Radiante. \\ Para los griegos, Brillante \\ Para los ouichuas, Resplandeciente.}

La constelación ha conservado en la civilización occidental, a través de las edades, el primitivo nombre que le dieran los egipcios: Can Mayor o sea Perro Grande, foorque çu formaosügiere a primera vista la idea de un cuadrúpedo. Por la misma razón, los quichuas vieron en ella, sin gran esfuerzo de imaginación, la figura de una llama.

Ya no cabe pues la menor duda de que Urcuchillay es Sirio con la constelación el Can Mayor a la que pertenece.

Pero se objetará ¿cómo ha podido producirse la confusión, el cambio de Sirio por Vega (Lyrae) cuando nadie se ocupa del primero y en cambio todos hablan de "la estrella que los astrólogos llaman Lyra"?

La confusión es muy sencilla y vamos a demostrar en quién, por qué y cómo se porduio.

El Licencilado Polo de Ondegardo, que ya se encontraba en el Perú en el año 1545, tué el primero que recogió directamente de boca de los indios los nombres de las estrellas o constelaciones a las que rendían culto, en las averiguaciones que hizo para descubrir "Los Errores y Supersticiones de los Indios". Sus investigaciones no tenían, en absoluto, carácter científico ni mucho menos astronómico. Ellas estaban encaminadas exc'lusivamente a descubrir huacas, conopas y en ge- 
neral todas las cosas que servían a los indios como ídolos u objetos de culto y veneración, para destruirlos materialmente o desterrarlos de su :maginación.

En la época en que el sacerdote Juan Polo de Ondegardo hizo sus estudios supeiiorés y recibió su licenciatura en la Universidad de Salamanca, a principios del siglo XVI, las ideas reinantes en los centros intelectuales europeos eran completamente rudimentarias $\circ$ absurdas en lo que se refiere a $\underline{\Lambda}$ stronomía, que generalmente era confundida con la Astrología. Se puede decir que la Astronomía propiamente dicha era completamente desconocida en esos centros. El estudio de esta ciencia estaba proscrito del mundo católico, que consideraba como charlatanes, hechiceros o brujos a los que se dedicaban a él, a los Astrólogos, como se les designaba cornúnmente. Estas creencias estaban más arraigadas aún en España, que si en algo contribuyó al adelanto de la Astronomía no fué precisamerte por intermedio de un católico sino, en época muy anterior, por el judío Abraham Azaquiel, con sus Tablas Toledanas (li80) y por los moros Alcabituis y Abu Ragel que bajo los auspicios de Alfonso X, el Sakic, calcularon las Tablas Alfonsinas (1252). Como exponente de la mentalidad de esa época está el Decreto del Santo Oficio del 15 de marzo de 1615 poniendo en el Index la magistral obra de Copérnico "De Revolutionibus", en la que demostraba el movimiento de la Tierra alrededor del Sol y e! del 23 de junio de 1633, condenando a Galileo $\mathrm{y}$ poniendo al Index sus "Dialogos", obra de divulgación del sistema de Copérnico.

Salido de este medio de fanatismo intransigente, el Licenciado Polo de Ondegardo. no tenía seguramente más que las ideas rudimentarias de Cosmografía y de Cosmogonía que estaban en auge para discutír con los peripatéticos la concordancia entre el sistema de Tolomeo y las Sagradas Escrituras. Sus conocimientos de los Astros no habían pasado más allá de la satisfacción de una muy natural curiosidad por saber el nombre de la más hermosa estrella que atraviesa el cielo del hemisferio Bs:eal en su mayor extensión. El la había visto culminar al sur del zeiit sobre el horizonte de Salamanca; se le ha grabado en ia memoria por ser la más brillante y por su color blanco azulado que is destaca sobre todas las que se ven en el cielo de Salamanca; ha preguntado pis su nnmbre probablemente a otro estudiante de teología - a un maestio, tan ignorantes como él en esta materia, y le han informado, casi a hurtadillas, que a esa hermosa estrella le llamaban Lira los astrólogos, los réprobos, aquellos con quienes no se puede tener contacto $\sin$ mancharse espiritualmente. $Y$ éste es todo el bagage de 
conocimientos astronómicos con que el Licenciado Polo de Ondegardo vino al Perú, y como él, todos los rionistas que vinieron antes y después de él. Información de lego, ni siquiera de diletanti, que él jamás trató de corroborar o enmendar, Información errónea en absoluto porque Lira ha sido y es el nombre de una constelación y no de una es-
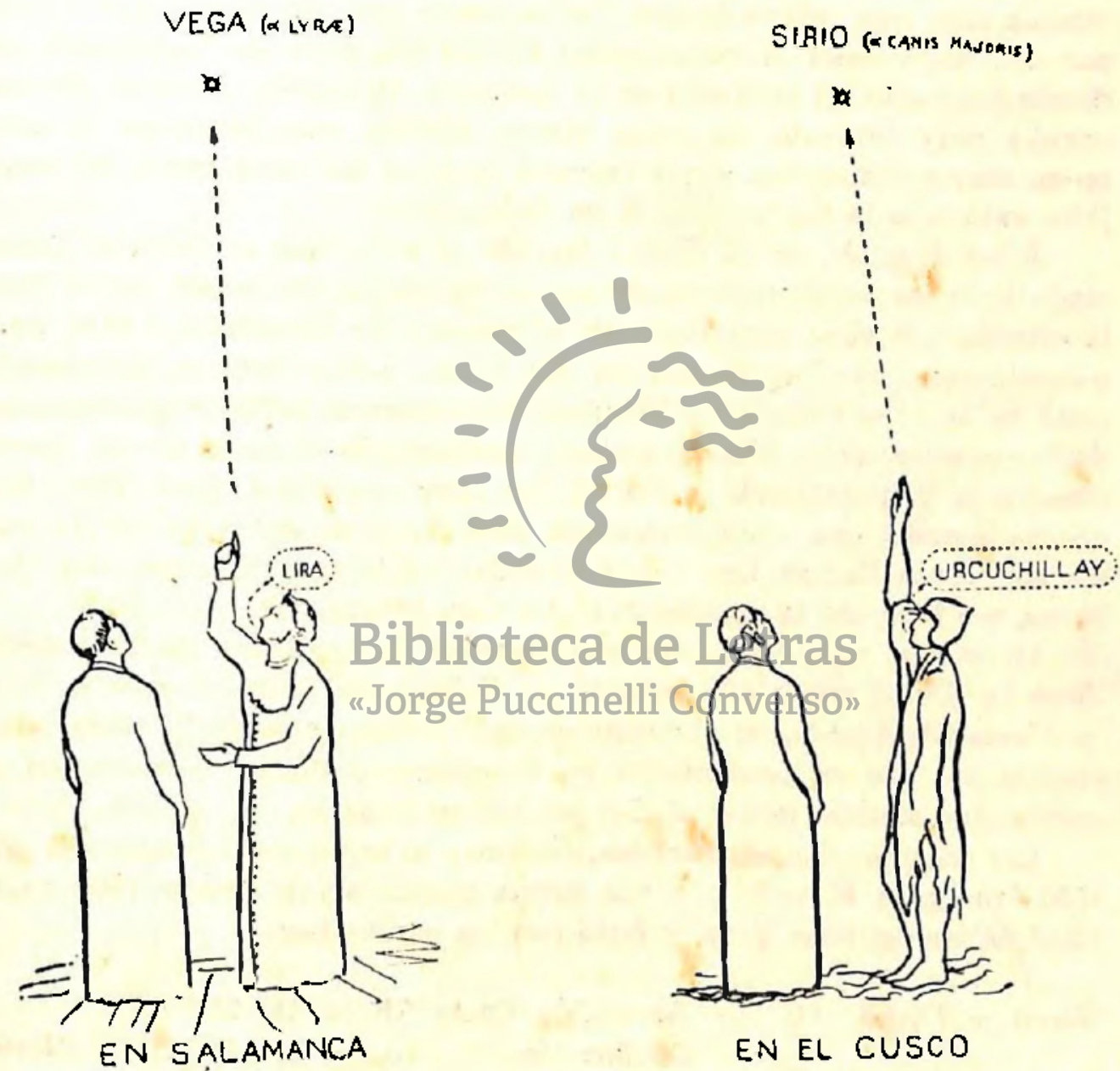

EN EL CUSCO

Fig. 4

trella, porque la estrella más brillante de esta constelación y la más hermosa del hemisterio boreal se ina llamado y se llama Vega y no Lira. (Fig. 4).

En el párrafo pertinente de su cbra "Los errores y supersticiones de los indios", el Licenciado Polo dice textualmente: 
"Y así los ovejeros hacían veneración a una estrella que ellos llamaban Urcuchillay, y que dicen es un carnero de muchos colores, el cual entiende en la conservación del ganado y se entiende ser la que los astrólogos llaman Iira".

Como se ve, aquí se trata de una estrella y no de una constelación, y Polo se guarda mly bierı de decir que él conose el nombre de esa estrella sino que refiere lo que "se entiende ser", el rumor que circula por ahí, tanto entre el vulgo como $\in \mathrm{n}$ los claustros de Salamanca, de donde ha traído tal recuerdo en la memoria. Recuerdo concreto de una estrella muy brillants, de color blanco azulado que atraviesa el cielo en su mayor dimensión y que culmina hacia el Sur muy cerca del zenit. Pero todo esto lo había visto él en Salamanca.

Años despuér. en el Cusco, cuando el indio que relataba al Licenciado Polo las supersticiones de sus antepasados, le señaló en el cielo la estrella que ellos adoraban bajo el nombre de Urcuchillay, éste, muy naturalmente, creyó reconocer en ell:a la que había visto en Salamanca, pues se le presentaba bajo idénticas condiciones: brillo y dirnensiones deslumbrantes, color blanco azulado, atravesando el cielo en su mayor dimensión y culminando hacia el Sur muy cerca del zenit. Pero ésta no era aquélla que dubitativamente dice "que se entiende ser la que los astrólogos llamar: Lira"; esta estrella era la más hermosa del Universo, era Sirio de la constelación de! Can Mayor. (1)

En efecto, calculande las coordenadas horizontales de la estrella Vega ( $\propto$ Lirae) sobréejghorizonien dei Salamanca) y de la estrella Sirio ( $\propto$ Canis Majoris) sobre el horizonte del Cusco, veremos la idéntica situación en que se presentaron en el momento de su culminación al observador situado frente al Sur en ambos lugares, en el año 1550.

Las coordenadas ecuatoriales, referidas al equinoccio medio del año 1550 (que para e! caso que nos ocupa puede servir desde 1500 hasta 1600) de las estrellas Vega y Sirio son las siguientes:

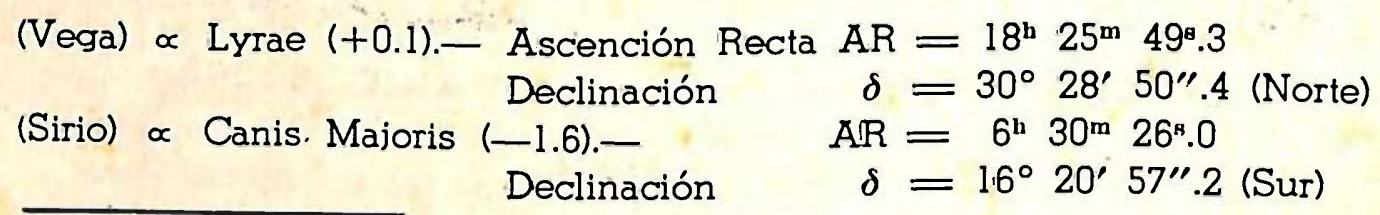

(1) Sirio y Vega pertenecen al mismo tipo espectral: el I en la ordenación de Sechi, - el A de la clasificación de Drapere, denominado tipo Siriano. cuyas características son: temperatura vecina a los $10,000^{\circ}$, color blanco azulado, rayas intensas de hidrógeno. 
La latitud de Salamanca es $\phi=+40^{\circ} 58^{\prime}$ (Norte)

La latitud del Cusco es $\quad \phi=-13^{\circ} 31^{\prime} 40^{\prime \prime} .0$ (Sur)

Las distancias zenitales meridianas de Vega y Sirio, observadas desde Salamanca y Cusco respectivamente y calculadas con la fórmula conocida $\mathrm{z}=\phi-\delta$, son:

En Salamanca

(Vega)
En el Cusco

(Sirio)

$$
\begin{array}{llll}
\text { (Latitud) } & \phi=+40^{\circ} 58^{\prime} & \text { (Latitud) } & \phi=-13^{\circ} 32^{\prime} \\
\text { (Declinación) } & \delta=+38^{\circ} 29^{\prime} & \text { (Declinación) } & \delta=-16^{\circ} 21^{\prime} \\
& z=+2^{\circ} 22^{\prime} \text { (Sur) } & z=+2^{\circ} 49^{\prime} \text { (Sur) }
\end{array}
$$

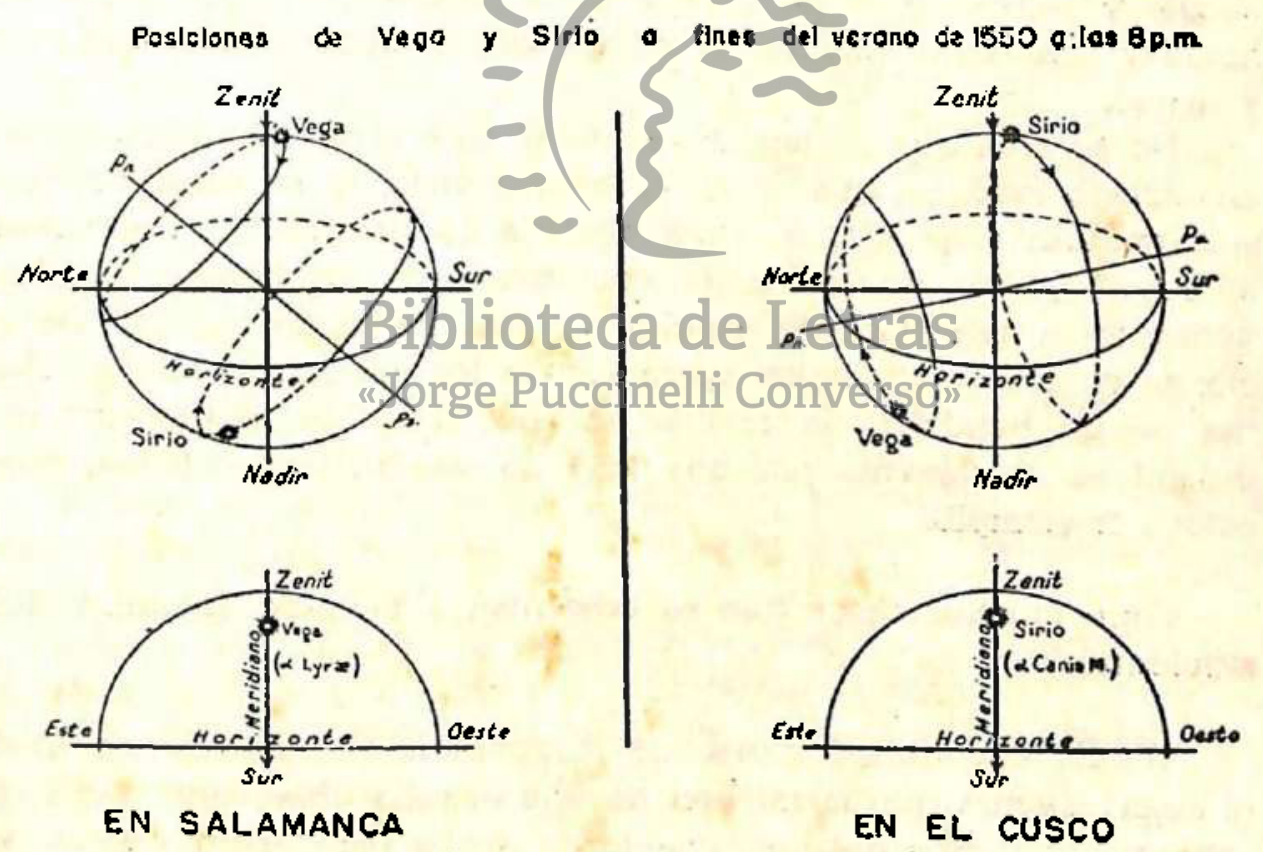

Fig. 5

La pequeña diferencia de $20^{\prime}$ en las distancias zenitales es imperceptible a simole vista. El diagrama adjunto da. una idea clara de esta identidad de situsiriones aparentes (Fig. 5). 
Hay adernás otras circunstancias que pueden haber contribuído a esta confusión del Lícenciado Polo:

li-La posición de Vega en la esfera celeste, está casi diametralmente opuesta a la de Sirio, pues las ascensiones rectas de ambas difieren en cerca de 12 horas $\left(180^{\circ}\right)$ de manera que el día de una, (que es cuando está sobre el horizonte) corresponde a la noche de la otra, (que es cuancic está jor debajo del horizonte). Así, cuando Polo vió a Vega to Lira como él la llamaba) sobre el horizonte de Salamanca, no riabía en todo el ciele otra estrella igual en color ni en brillo; y cuando vió a Sirio sobre el horizonte del Cusco, tamposo había en el cielo otra estrella semejante en color y en magnitud. Se puede asegurar, por otra parte, que Pc'o iamás ha visto a Sirio desde Salamanca, porque esta estrella culmina allá en invierno y a una altura imuy baja sobre el horizonte $\left(32^{\circ}\right)$.

2 - - El Licenciado Polo ha visto, desde Salamanca, culminar a Vega en las primeras hcras de la noche al finalizar el verano de Europa, entre agosto y setiembie; y ha visto a Sirio, desde el Cusco, a las mismas horas y también al finalizar el verano del hemisferio Sur, entre febrero y marzo.

No se crea que es tan grave, en sí, este error de identificación del Licenciado Polo, ya que, como lo hemos visto, él no tenía por qué poseer grandes ni pequeños conocimientos de Astronomía. Equívocos tanto o más graves que estos los cometen hasta los hombres de ciencia dedicados a esta clase delestudios cuando tratan de explicar las alteraciones de los movinientos aparentes] de dos astros al pasar el observador de un hemisferic a otro, o cuando tratan de identificar un astro guiándose únicamente por una sola de sus particularidades, como el color por ejemplo.

Entre muchos cases que se presentan a menudo citaremos los dos siguientes:

19--En una obra francesa de Astronomía Geodésica, modernísima, el autor asegura que la imagen de una estrella observada hacia el Este atraviesa el campo del instrumento de arriba para abajo, cuando el observador se encuentra en el hemisferio Norte, y de abajo para arriba cuando él se encuentra en el hemisferio Sur. Lo absurdo de estia afirmación salta a la vista sin necesidad de ninguna demostración, pues, en ambos casos, el sentido de la marcha de las imágenes es siempre el mismo para los dos hemisferios. 
2 - - Hace algunos años, un profesor europeo, hombre de ciencia muy justamente considerado y apreciado, nos mostraba a varias personas el planeta Marta acercándose al zenit, y aseguraba que el astro en cuestión no padía ser otro que Marte dado el color rojo, propio de diaho planeta. Pues bien, el astro que nos mostraba el profesor no era tal planeta sino la estrella Antares de la constelación del Escorpión que tiene el mismo color de Marte, perc que se diferencia de él por el centelleo caracteristico de la la luz propia de todas las estrellas y en el cual no había reparado el ilustre profesor. Costó algún trabajo sacarlo de su ərror, pəro al fin tuvo que convencerse. Así pues, cualquiera, hasta los hombres de ciercia, pueden equivocarse y, con mayor razón, un lego, como lo era en esta materia el Licenciado Polo.

La confusión del Licenciado Polo no tendría gran importancia si sobre su lamentable error no se hubiera edificado casi todas las teorías de la astronomía incaica, si no hubiera inducido a los investigadores antiguos y modernos a buscar en la vecindad de Lira, o sea en el hemisferio boreal,. la identificación de las demás constelaciones conocidas por los primitivos peruanos dando rienda suelta a su fantasía y abandonando la región austral, donde realmente se encuentran todas las más imporiantes estrellas, conocidas y veneradas por los indios como lo veremos más adelante.

\section{Conclusión.}

I-El nombre Urcuchillay de los quichuas tiene" el mismo significado que Sothis de lns egipcios y que Seiroias de los griegos, significado derivado de la cualidad de brillantez y resplandor que en grado máximo caracteriza a una estrella única en el Universo, es decir a Sirio ( $\propto$ Canis Majoris).

2:-En la constelación formada por las estrellas circunvecinas, los astrónomos eyipcios y griegos vieron la forma de un perro grande; los quiohuas vieron, muy naturalmente, la forma de una llama y le dieron el nombre de la iestrelia más 'brillante.

Luego:

$$
\text { UR:CUCHILLAY = SIRIO, CON EL CAN MAYOR. }
$$

Todos los cronistas que se ocuparon de las constelaciones conocidas y adoradas por los antiguos peruanos, al referirse a la constelación Urcuchillay, repiten lo que dijo Juan Polo de Ondegardo: "así los ovejezos hacían veneración y sacrificios a una estrella que se llama Urcuahi- 
llay, que dicen es un carrero de muchos colores, el cual entiende en la conservación ciel ganado y se entiende ser la que los astrólogos llaman Lyra. $Y$ los mismos adoran a otras dos que andan cerca della que llaman Catuchillay, Urcuchillay que fingen ser una oveja con un cordero".

Los comentaristas que se han ocupado de los conocimientos astronómicos de los incas han buscado todos ellos a esta constelación "Catuchillay, Urcuahillay" de doble nombre, entre las constelaciones zodiacales, o como el señor Lehmann, en el hemisferio boreal en las proximidades de la constelaciór de la Lira.

No vamos a seguir las divagaciones del señor Lehmann que cree encontrar a Cátuchillay y Urcuchillay en las proximidades de la constelación de la Lira, entre las pequeñas estrellas del Cisne, por seguir la frase del cronista de que estas estrellas "andan cerca della" (La Lira); pues la figura que él iraza sería imposible identificarla en el firmamento por la razón de que toma estrellas muy pequeñas y porque, desde el Cusco, esta figura se vería invertida, es decir con las patas para arriba. No me parece necesario insistir en demostrar lo absurdo de esta afirmación del señor Lehmann.

La constelación Catuchillay Urcuchillay no está constituida efectivamente por una constelación de estrellas sino que la forman dos estrellas principales de la constelación del Centauro y una mancha negra dentro de la Vía Láctea queil partiendo de estas dos estrellas, va a terminar en ia constelación del Escorpión mancha negra que tiene efectivamente la forma de una llama a cuyos pies se puede imaginar fácilmente la forma de su cría. Se ve claramente que de las dos estrellas brillantes, alfa y beta del Centauro, pante una mancha delgada que semeja el cuello de la llama. ensanchándose después para formar el cuerpo, del que se desprende como ji-ones manahas negras que semejan las patas y la forma de la cría. Yo recuerdo haber oído de boca de los peones que cosechaban el maíz en el mes de mayo, que a aquellas manchas lás ilamaban'la llama y a las dos estrellas brillantes del Centauro los ojos de la llama.

Corroborando esta idea tenemos el relato de Garcilaso de la Vega: "en la Vía que los astrólogos llaman Láctea hay unas manchas negras que van por eila a lo largo, quisieron imaginar que había una figura de oveja, con su cuerpo entero que estaba amamantando a un cordero. A mí me la querían mostrar diciendo: ves acullá la del cordero mamando; ves el cuerpo brazo y piernas del uno y del otro; mas 
yo no veía las figuras sino las manchas y debe ser por no saberlas imaginar".

Ya no queda pl:ss duda de que Catuchillay Urcuchillay es la maincha de la Vía Láctea que se extiencle desde el Centauro hasta el Escorpión y que hasta ahora en algunos puntos de la sierra los indígenas la designan con el nombre de "la llama", así como a las dos estrellas principales dei Centauro con el de "los ojos de la llama". Los antiguos peruanos concentraban, seguramente, su atención en las dos estrellas principales, denominando Catuchillay a la mayor (alfa Centauro) y Utcuchillay a la menor (beta Centauro). No debemos sorprendernos que el nombre Urcuchillay esté adjudicado simultáneamente a dos estrellas, puesto que en el segundo caso, el nombre no está solo sino asoziado al de Catuchillay, que es la madre. Por otra parte, entre las constelaciones conocidas aciualmente, existen muchas con el nombre repetido, pero asociado con alguna circuntsancia, por ejemplo el Can Mayor y el Car Mienor, la Hidra macho y la Hidra hembra, la Osa Mayor y la Osa Menor, etc.

Partiendo de la errónea ubicación de Urcuchillay (Sirio), se ha buscado también en el hemisferio Norte la identificación de otras constelaciones tales como. Hulaucha (el nambre furioso), que el señor Lehmann pretende idenuificar con la Osa Mayor en una determinada posición que jamás llega a adoptar vista desde el Cusco; pues esta constelación boreal, muy próxima alpolel Norte aparefe en érma casi horizontal sobre el horizonte del Cus $\%$ y rapresenta más bien la forma que nosotros hemos denominado de una cacerola invertida. 